\title{
Cittadini d'Europa. Un progetto didattico per le nuove generazioni
}

\author{
di Paolo Battifora and Giosiana Carrara
}

Nov 19, 2019 | Didattica in classe $|\underline{0}|$

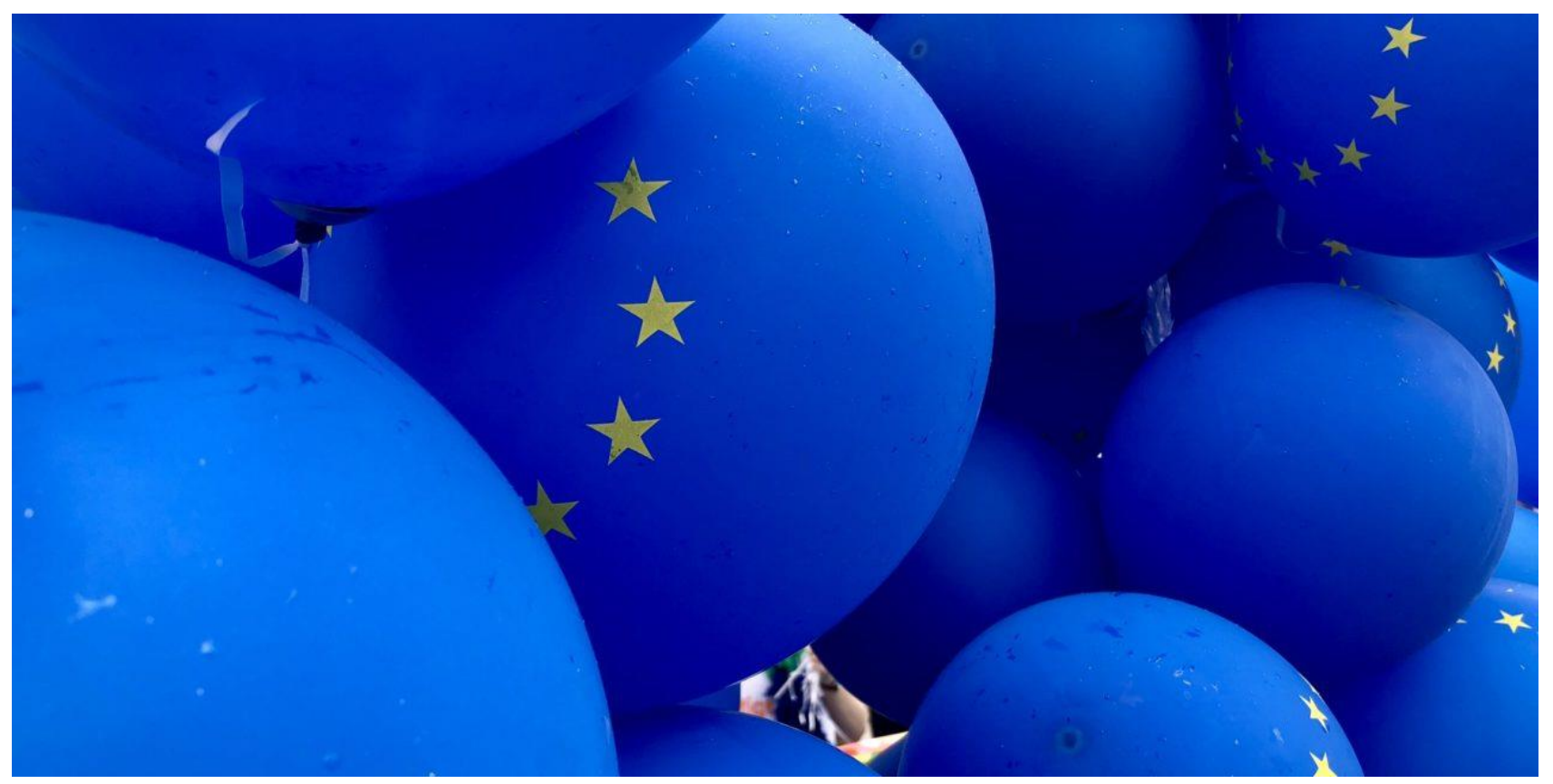

Palloncini blu con la bandiera dell'Unione europea - Foto di Jonatan Svensson Glad, scattata il 4 agosto 2018 e pubblicata su Flickr. Licenza CC 2.0.

\begin{abstract}
In una fase storica connotata dalle dinamiche della globalizzazione e da possenti fenomeni economici e sociali dai possibili effetti destabilizzanti, l'Unione Europea non gode di buona salute, venendo percepita quale farraginoso organismo burocratico e opaco centro di potere, lontano dalle quotidiane esigenze dei cittadini. Grazie anche all'operato di politici e mezzi di informazione abili nell'alimentare risentimenti e senso di insicurezza collettivi, funzionali alla propaganda populista e sovranista, l'Europa sempre più viene additata come capro espiatorio di ogni problema e criticità. L'ambizioso progetto didattico "Memoria storica, cittadinanza, innovazione civica. Progetto per le nuove generazioni”, realizzato nel 2019 dall'Istituto ligure per la storia della Resistenza e dell'età contemporanea "Raimondo Ricci" (ILSREC) e rivolto agli studenti degli istituti superiori della Liguria, tramite una coinvolgente metodologia interdisciplinare, in grado di coniugare momenti formativi frontali, attività seminariali, giochi di ruolo, discussioni comuni, quiz, ha promosso nelle scuole i temi della cittadinanza ed integrazione europea, coniugando storia, diritto e lucida lettura dell'attualità.
\end{abstract}

Paolo Battifora ha scritto i primi tre paragrafi e Giosiana Carrara i restanti. 
Tutta colpa dell'Europa. Lo si sente dire in giro, lo si legge sui giornali, lo si ascolta nelle reprimende di politici in cerca di facile consenso e in opinionisti dalla decisa verve polemica. Per non parlare dei social media, dove è tutto un profluvio di invettive e strali a senso unico sugli organismi di Bruxelles. Da diversi anni l'Unione Europea mostra di non godere di buona salute: sottoposta ad attacchi sempre più mirati e concentrici, essa è soggetta a un processo di crescente delegittimazione, foriero di possibili effetti esiziali. Dare la colpa all'Europa per tutto ciò che non funziona, va a rilento o non soddisfa le aspettative, più o meno fondate e legittime, di questa $o$ quella nazione, ceto sociale, formazione politica, sembra essere divenuto il comodo alibi, adottato da qualsiasi soggetto collettivo, per sgravarsi di ogni responsabilità in merito alle proprie scelte, ritardi, inazioni, e per ovviare a seri esami di coscienza, suscettibili di creare profondo imbarazzo. Il mantra "ce lo chiede 1'Europa", "ce lo impone Bruxelles", "non siamo più padroni a casa nostra", ignora - o finge di ignorare - che l'Europa siamo anche noi e che le decisioni prese in sede comunitaria, lungi dal configurarsi quali diktat imperiosi, sono anche il frutto del consenso formulato, a vari livelli, dai nostri rappresentanti, troppo spesso affetti da sindromi amnesiche una volta valicate le Alpi e fatto ritorno sul patrio suolo.

Decisamente comodo, e al passo coi tempi, il sistematico ricorso a un capro espiatorio avente le sembianze di una torva Unione, sinonimo, tout-court, di carrozzone burocratico, algida tecnocrazia nemica dei popoli, realtà lontana e indifferente ai bisogni e ai problemi quotidiani della gente comune. Emblematica, in proposito, una vignetta satirica in cui un bambino, timoroso dei prevedibili rimbrotti materni per aver rotto in casa un vaso con il pallone, pensa bene di ricorrere a un ormai collaudato espediente, prorompendo in un perentorio ' $E$ ' stata l'Europa! E' andata da quella parte!'[1]. L'Unione Europea, insomma, come facile bersaglio, quanto mai funzionale alle mire elettorali e alle logiche populiste di politici adusi allo sfruttamento dei timori e risentimenti collettivi e decisamente privi di scrupoli nel fomentare pulsioni e dinamiche potenzialmente deleteri per la tenuta stessa delle istituzioni democratiche e della convivenza civile[2].

\section{Giano bifronte?}

Risulta evidente come un tale "sentire", seppur non generalizzabile e attribuibile a tutte le componenti e articolazioni della società italiana, influisca (e non poco) sulla percezione dell'Unione europea e degli organismi comunitari da parte delle nuove generazioni: nati in un periodo storico di pace e benessere, valutazione che nessuna considerazione critica sull'attuale congiuntura economica e realtà sociale può seriamente mettere in discussione, i giovani sono poco portati a riflettere sulla storia della prima metà del Novecento e a cogliere, di conseguenza, la radicale novità e importanza costituita da un progetto sovranazionale che, iniziato negli anni Cinquanta, avrebbe posto fine a secolari rivalità e tensioni, creando le premesse di quel percorso che, passo dopo passo, trattato dopo trattato, avrebbe condotto il continente all'attuale realtà di 28 stati membri - in attesa del tormentato compimento della Brexit - della UE[3].

Ritenuta per certi versi come ovvia e scontata, come una realtà ormai imprescindibile e facente parte del proprio vissuto e abituale orizzonte esperienziale - basti pensare, per fare alcuni esempi, al progetto Erasmus in ambito universitario, alla moneta unica, alle opportunità formative e di lavoro, agli scambi culturali, alle frequentazioni sociali -, l'Europa, sorta di Giano bifronte, rischia di venir colta da molti giovani come arcigna matrigna, arida contabile, opaco centro di potere funzionale agli interessi di banche, borse e settori finanziari, artificiale creatura priva di una salda e definita "identità"[4], freddo organismo confliggente con il "naturale" sentimento di appartenenza nazionale[5] e addirittura incombente minaccia nei confronti di una sovranità avvertita a rischio di progressiva erosione[6]. 


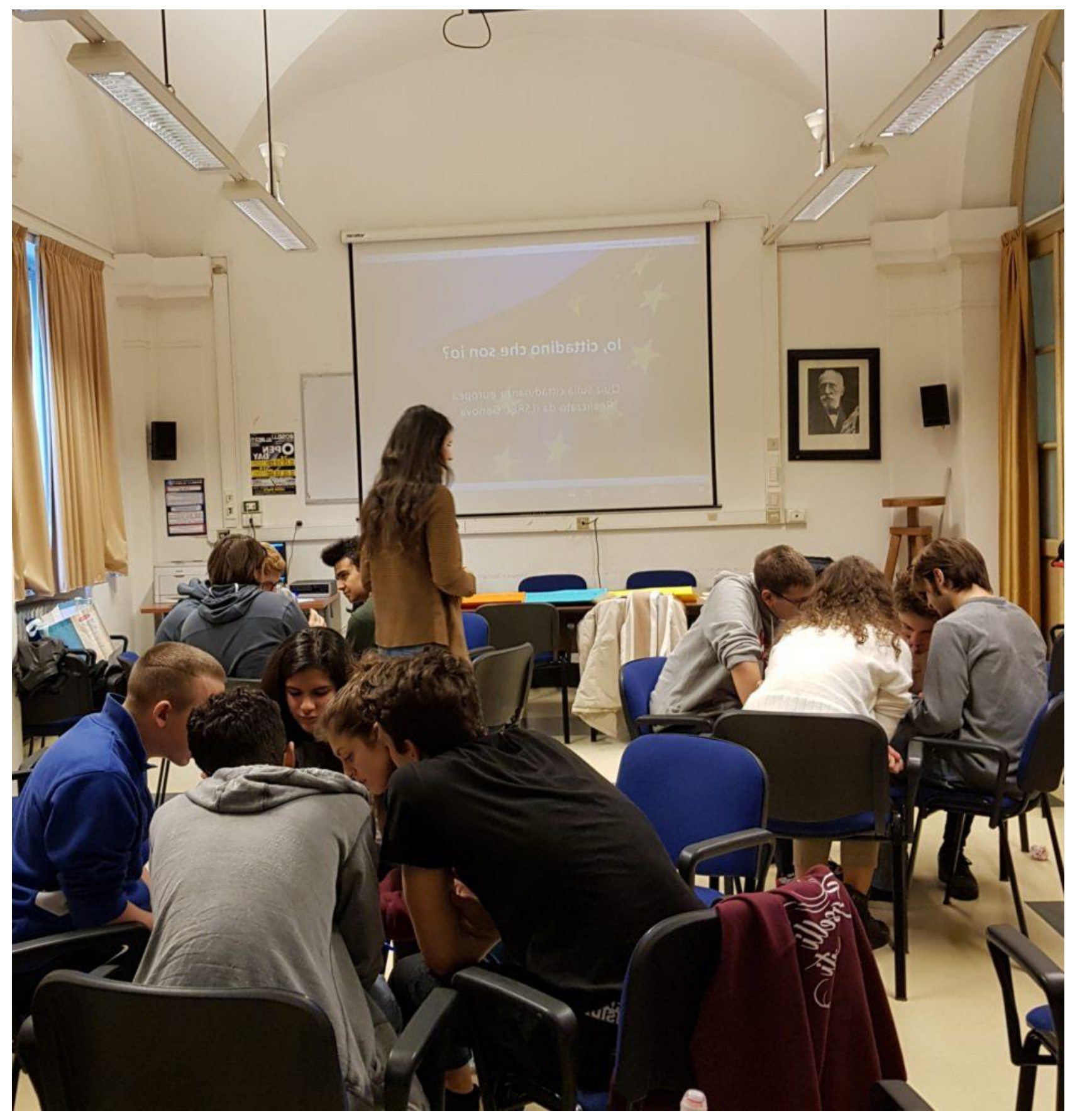

\section{Il progetto didattico}

Lungi dal voler indulgere in atteggiamenti apologetici "a prescindere" nei confronti della UE o dall'intento di mascherare, sottovalutare, minimizzare le innegabili manchevolezze, ritardi, deficit di democraticità che ancora, sotto molti punti di vista, connotano molte procedure decisionali degli organismi comunitari, l'Istituto ligure per la storia della Resistenza e dell'età contemporanea "Raimondo Ricci" (ILSREC) ha varato un progetto che, saldando l'orizzonte nazionale con quello europeo, potesse contribuire alla formazione di una matura e consapevole coscienza civile degli studenti delle scuole superiori della Liguria. "Memoria storica, cittadinanza, innovazione civica. Progetto per le nuove generazioni" è il titolo dell'iniziativa didattica che l'ILSREC ha portato nelle scuole per promuovere i temi della cittadinanza ed integrazione europea, dei diritti universali, della democrazia, della tolleranza, del nesso tra conoscenza storica e lettura dell'attualità. Il progetto è nato dall'esigenza di confrontarsi con le nuove sfide poste dall'età della globalizzazione, epoca connotata da migrazioni di massa, mutamenti climatici, tensioni innescate in varie aree del globo da 
conflitti, guerre civili, fondamentalismi religiosi, mutamenti negli assetti geopolitici mondiali, trasformazioni in senso multietnico delle società, rivoluzioni tecnologiche, crisi economiche, disorientamenti identitari, disagi psicologici e sociali a fronte di repentini cambiamenti e al cospetto di un futuro di ardua decifrazione.

Promosso e realizzato in collaborazione con l'Ufficio scolastico regionale per la Liguria, l’Università di Genova-Dipartimento di Scienze Politiche e altri enti e istituzioni[7], "Memoria storica, cittadinanza, innovazione civica. Progetto per le nuove generazioni" si è rivolto agli studenti delle scuole superiori e agli universitari, adottando una metodologia didattica interdisciplinare, mirata a coinvolgere attivamente i partecipanti e in grado di coniugare momenti formativi frontali, attività seminariali, giochi di ruolo, discussioni comuni, quiz: un procedimento idoneo a creare un adeguato spazio per la trattazione e l'approfondimento di temi e questioni inerenti la storia contemporanea, il diritto comunitario, il diritto costituzionale, la geopolitica, l'economia, la sociologia, il pensiero europeista.

Articolato nell'arco temporale gennaio-giugno 2019, il progetto ha coinvolto - tra licei classici, scientifici, artistici e istituti di istruzione superiore - sette scuole di Genova, tre di Savona e provincia, una della provincia di Imperia. Gli studenti della scuola che hanno preso parte complessivamente alle attività laboratoriali sono stati oltre 1.900 e 39 i docenti che hanno aderito alla proposta didattica dell'ILSREC[8].

Non limitato al solo mondo della scuola, l'impegno si è rivolto anche alla società civile, per la quale sono stati organizzati a Genova molteplici eventi e iniziative collaterali, seguite con interesse da circa 1.200 cittadini. Di particolare rilievo sono stati il convegno internazionale "Visions of Europe in the Resistance: Figures, Projects, Networks, Ideals"[9], la mostra artistica "La nuova Europa: un nuovo umanesimo tra arte e memoria" e relativa cerimonia pubblica di premiazione dei vincitori[10], la presentazione del numero monografico 2/2019 della rivista dell'ILSREC “Storia e memoria", intitolata Dove va l'Europa?[11].

Nel novero delle iniziative[12], da segnalare ancora la conferenza-dibattito "L'ideale europeista nel secolo dei totalitarismi", che il 9 maggio, in occasione della Festa dell'Europa, lo storico Marcello Flores ha tenuto a Palazzo Ducale di fronte ad oltre 400 studenti[13] e nel corso della quale il Presidente dell'ILSREC Giacomo Ronzitti ha consegnato gli attestati di partecipazione al progetto didattico ai docenti delle classi coinvolte. 


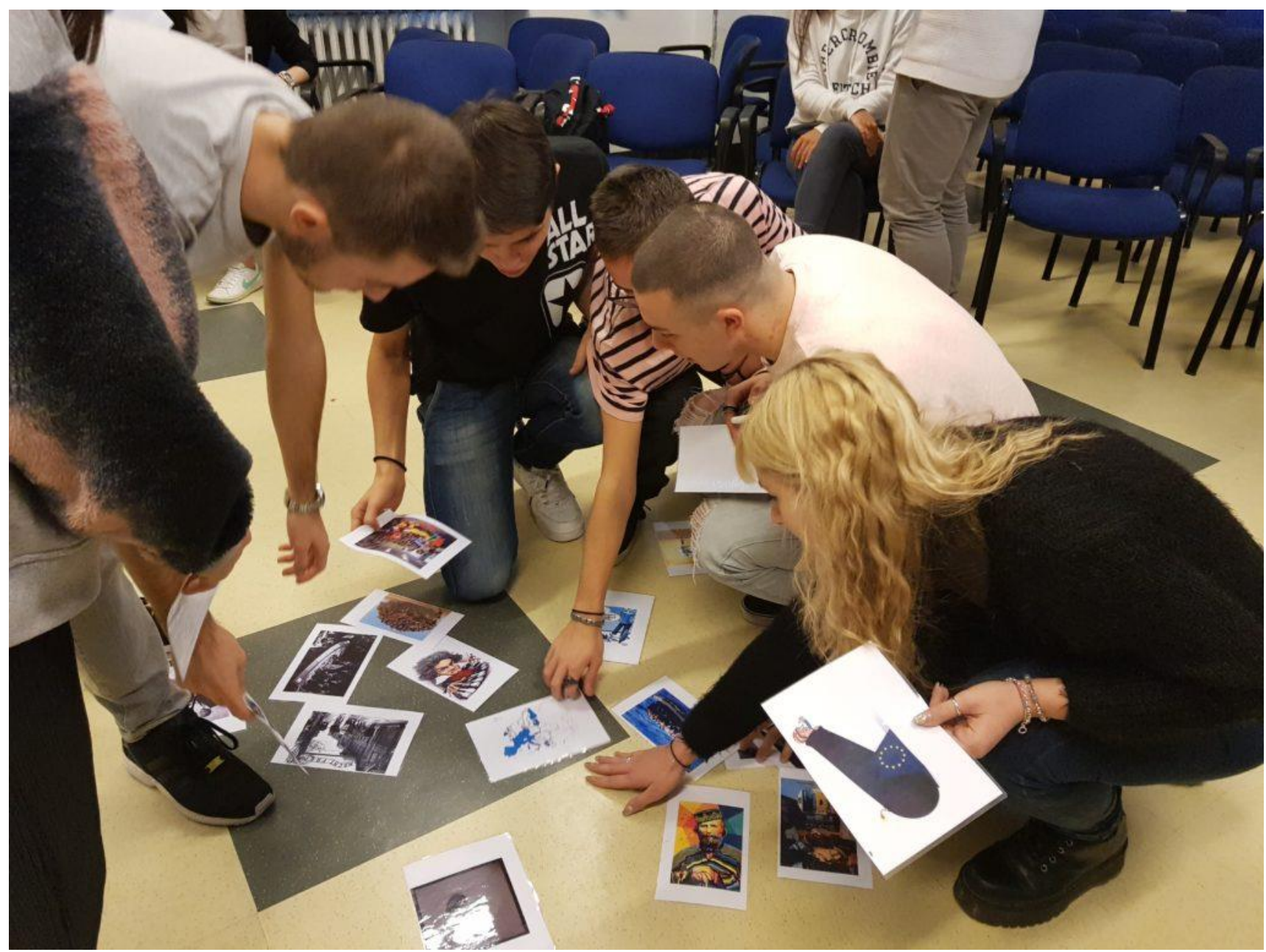

\section{Un percorso articolato}

In seguito ad un'ampia e diffusa presentazione a livello regionale, centinaia di giovani delle province di Genova, Savona e Imperia sono stati coinvolti nei laboratori interdisciplinari sul tema della cittadinanza europea, ideati da Angelica Radicchi con la collaborazione di Silvia Giulini. L'imponente azione educativa è stata realizzata, di volta in volta, da due diversi formatori dello staff dell'ILSREC[14], che hanno operato con gruppi classe composti da una media di 25 alunni e alla presenza di almeno un insegnante referente.

Si è cercato di porre i giovani, con i loro bisogni e le loro curiosità, al centro dell'attività formativa. Per questo, tanto i singoli moduli proposti nelle scuole del triennio (discussioni, quiz, gioco di ruoli, partecipazione al Concorso d'arte "La nuova Europa: un nuovo umanesimo tra arte e memoria", ecc.) quanto le esperienze di comunicazione storico-didattica di tipo tradizionale rivolte agli studenti medi e universitari (attività seminariali, incontri formativi frontali, conferenze-dibattito con personalità del mondo politico, accademico e associativo e visita alle mostre allestite in occasione degli eventi correlati al progetto) hanno fatto leva su metodologie didattiche avanzate e innovative. Si è scommesso sulla possibilità di coinvolgere in prima persona le giovani generazioni, predisponendo le condizioni per affrontare temi complessi inerenti alla storia contemporanea, come la geopolitica, il diritto costituzionale e comunitario e il pensiero europeista.

L'obiettivo ha impegnato per sei mesi lo staff che dalle città capoluogo delle province liguri ha raggiunto le periferie per incontrare ragazze e ragazzi provenienti da licei, istituti tecnici e scuole professionali. Al termine, i commenti dei gruppi docente-classi e i questionari di verifica e 
valutazione finale, nella stragrande maggioranza dei casi, hanno confermato che le buone pratiche messe in atto, sostenute dallo sforzo di creare un ambiente di apprendimento proficuo e stimolante, hanno consentito di ottenere i risultati attesi ma non scontati.

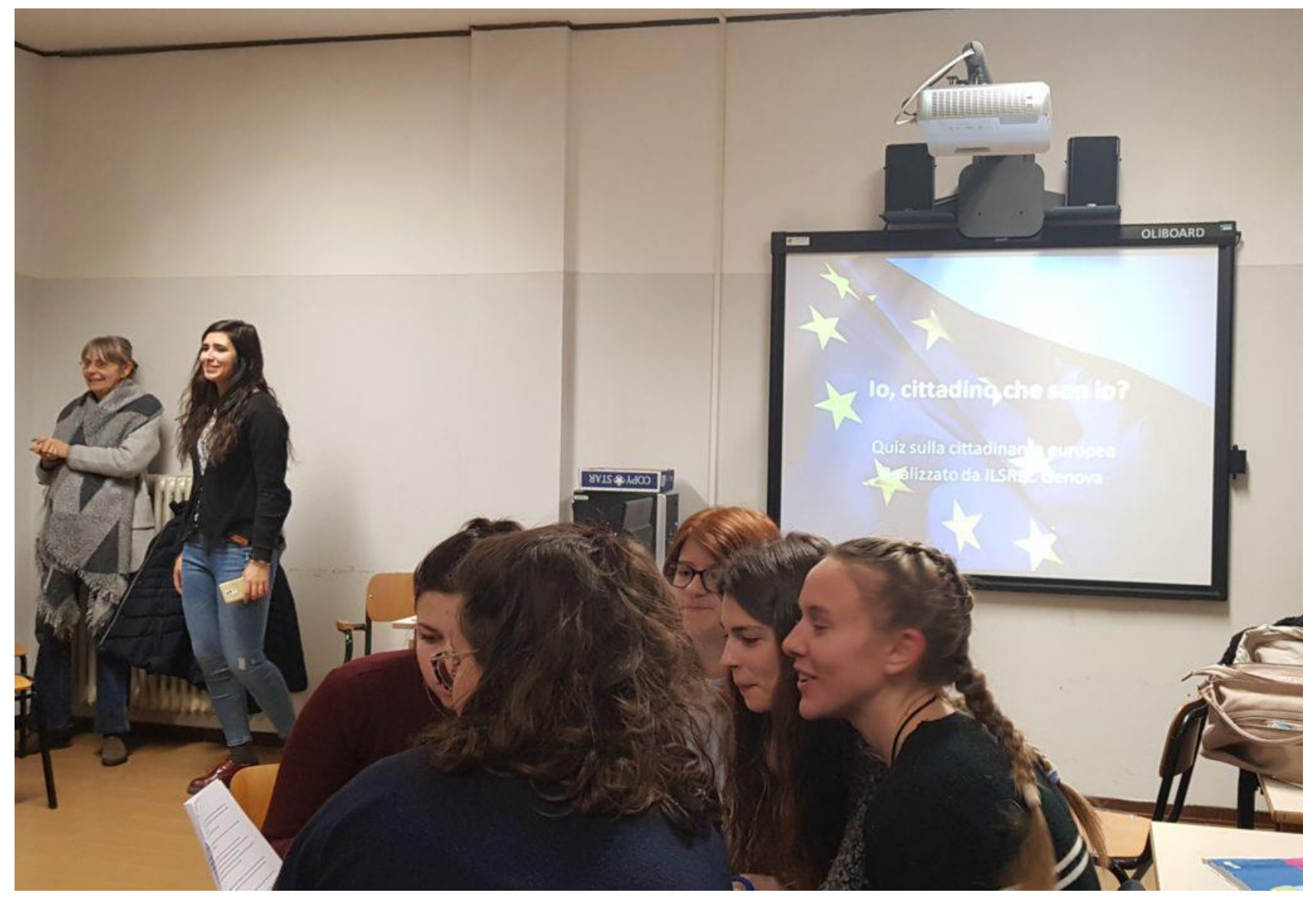

\section{Il primo modulo}

I laboratori di cittadinanza europea proposti ai docenti e agli alunni del triennio delle superiori hanno avuto una durata complessiva di 4 ore per classe, suddivise in due moduli di 2 ore ciascuno, di cui il primo presentava un livello teorico e tecnico più complesso mentre il secondo risultava orientato ad attività di gruppo ispirate alla didattica ludica e al cooperative learning. I contenuti generali hanno fatto leva sulle conoscenze di base dell'idea di Europa, i processi di integrazione all'Unione europea, le istituzioni e il diritto dell'UE ("Pillole di storia" e "Pillole di diritto"), con particolare riferimento ai gruppi politici in vista delle elezioni del maggio 2019[15] e al concetto di cittadinanza europea curvato sull'attualità. Ai docenti è stato chiesto di scegliere la combinazione di moduli ritenuta più adatta alla fisionomia della classe e alle sue conoscenze pregresse. In linea di massima, gli oltre 1.900 studenti del triennio e i 39 docenti, che hanno partecipato alle attività laboratoriali nelle tre province di Genova, Savona e Imperia, hanno aderito alla proposta didattica svolgendo entrambi i moduli.

Il primo modulo, della durata complessiva di 2 ore, ha previsto due attività, "Ice breaker" e "Pillole di storia", rispettivamente di 40 e 20 minuti, seguite da tre azioni: "This time I'm voting", "Questo o quello?" e "Pillole di diritto" di 20 minuti ciascuna. È parso prioritario ricorrere a strategie di apprendimento partecipate, diversificare la trattazione degli argomenti ed alternare le azioni didattiche in modo da variare il più possibile tempi e modalità di azione. 
Il progetto ha avuto inizio con l'“Ice breaker" ("rottura del ghiaccio"). La prima richiesta consisteva nel disporre di un'aula ampia, dotata di un computer collegato a una lavagna interattiva multimediale, in cui fosse possibile destrutturare lo spazio tradizionale per creare un ambiente adeguato ad attività anche di tipo motorio. Si è quindi proposto al gruppo docente-classe di posizionare i banchi lungo le pareti e si è chiesto agli alunni di disporsi in cerchio. Sono state collocate sul pavimento 32 immagini plastificate raffiguranti alcune tra le più note questioni pertinenti all'Europa: foto e disegni riferiti a temi di attualità (crisi economica e migratoria, introduzione dell'Euro, questione Brexit), a momenti storici (personalità politiche del passato e del presente, firma dei Trattati di Roma, ecc.) e alla libertà di movimento (indicazioni di confini tra gli Stati, esempi di multiculturalismo o di esperienze Erasmus) e carte del continente con la rappresentazione dei Paesi aderenti all'Unione. I ragazzi venivano sollecitati a selezionare un paio di immagini e a presentarsi secondo un ordine dato, illustrando in breve le ragioni delle proprie scelte.

Tramite una prima ricognizione, s'intendeva far emergere le opinioni sull'Europa più diffuse tra $i$ giovani a partire da un contesto non giudicante e in modo che il gruppo-classe, allentata la tensione e le difese generalmente sottotraccia nel tempo della lezione, potesse dialogare con agio e naturalezza. Gli studenti, dapprima incerti sull'atteggiamento da assumere (alcuni mantenevano la compostezza del tempo-lezione, altri erano tentati dalla "rottura delle righe" dell'intervallo), presto abbandonavano i clichés atteggiandosi in modo autonomo, specie dal momento in cui - chiamati a presentarsi in prima persona rispettando i tempi di esposizione dei compagni - esprimevano le loro considerazioni.

Nell'azione complementare ("Pillole di storia") il formatore, raccolte le opinioni dei ragazzi sull'UE e scelte a sua volta 2 immagini, le commentava introducendo alcune conoscenze di base riferite all'idea di Europa e al processo di integrazione europea[16]. Il breve excursus storico, curvato a seconda dell'età dei ragazzi, delle domande poste e delle conoscenze pregresse, si concludeva con cenni alle prime elezioni europee del 1979, così da porre i fondamenti per gli argomenti successivi. Nella sostanza, si presentavano gli elementi costitutivi degli organismi dell'Unione europea e le ragioni storico-politiche ed economiche che portarono alla sua istituzione.

\section{“This time I'm voting" e "Questo o quello?"}

L'attività successiva, "This time I'm voting", tratta dalla campagna lanciata dal Parlamento europeo (PE) per le elezioni del maggio 2019, prevedeva la proiezione di brevi video esplicativi dell'iter elettorale e di infografiche relative ai partiti politici italiani e ai rispettivi gruppi politici europei nonché all'attuale ripartizione del PE.

La lezione partecipata, intercalata da questioni volte a rafforzare la comprensione dei contenuti e da frequenti pause per sollecitare interventi di chiarificazione, veniva completata dall'attività "Questo o quello?" Al centro dello spazio-aula si ponevano fogli di carta in formato A3 di diversi colori, ciascuno dei quali era denominato da una coppia di opzioni o di alternative possibili inerenti a un problema europeo come, ad esempio, sovranità nazionale / maggiore integrazione europea, sicurezza e protezione dei confini / politiche di accoglienza, Euro sì / Euro no. Ogni studente aveva la consegna di annotare - tramite il segno X - l'opzione ritenuta preferibile. Al termine, raccolti i fogli, si discutevano i risultati. Spesso la classe, dividendosi in due gruppi rappresentativi delle opinioni opposte, metteva in atto uno spontaneo debate. In questi casi il formatore, affidandosi alle indicazioni della didattica controversiale, gestiva la discussione avendo ben chiari due importanti scopi: da un lato, garantire la partecipazione democratica dei sostenitori tanto del pro-Europa quanto del contra, dall'altro, indurre ciascuno a ponderare le posizioni sostenute e a valutarne sempre le conseguenze. Talora capitava che gruppi di ragazzi, collocatisi in una posizione mediana 
tra le due opzioni indicate, vi si trincerassero "paludandosi". Si sollecitava pertanto chi aveva assunto questo atteggiamento a soffermarsi su singoli casi che esemplificavano il tema generale discusso. In altre parole, si procedeva induttivamente, affinché la concretezza delle questioni inducesse chi appariva meno coinvolto nel dibattito a esprimere un più netto parere. In generale, proprio l'intervento a più voci dell'improvvisata assemblea di classe permetteva di focalizzare, in proiezione, la complessità delle scelte del Parlamento europeo - sia in termini di principio sia di responsabilità operative - alla base delle opzioni date.

Il primo modulo si concludeva con un intervento di tipo frontale ("Pillole di diritto"), riassuntivo del funzionamento e dei poteri del Parlamento europeo presentato con l'ausilio di slide e brevi video[17].

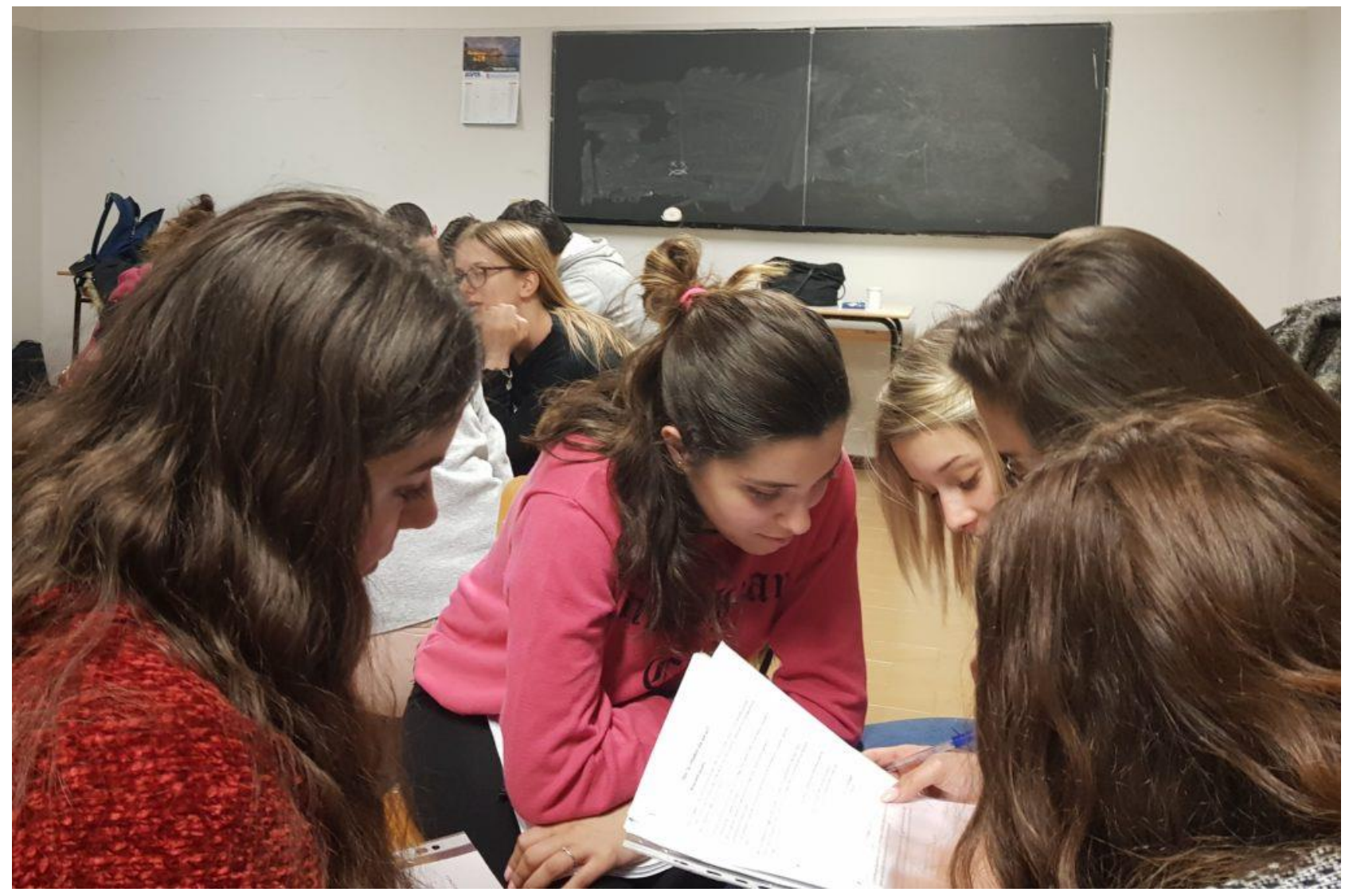

\section{Il secondo modulo}

Il secondo modulo, attivato a distanza di una settimana circa dal primo, era generalmente preceduto dal ripasso che l'insegnante referente aveva svolto nella classe sui temi già affrontati avvalendosi del kit di materiali illustrativi forniti[18].

La visione di un Powerpoint, consentiva di riepilogare le funzioni delle principali istituzioni europee (Consiglio europeo, Consiglio dell'UE, Parlamento europeo, Commissione europea e Corte di giustizia) e dei poteri corrispondenti (legislativo, esecutivo e giudiziario). Di fatto, il modulo prevedeva due azioni distinte, il dibattito "Vero o falso" e il quiz "Io, cittadino che son io?" della durata di 50 minuti ciascuna, e si concludeva con il "Feedback". In quest'ultima azione, i ragazzi erano invitati a scrivere in forma anonima su post-it brevi valutazioni del progetto e a posizionarle in corrispondenza di tre cartelloni intestati con le diciture "cosa mi è piaciuto", "cosa non mi è piaciuto" e "cosa cambierei". 


\section{"Vero o falso?" e "Io, cittadino che son io?"}

Il dibattito "Vero o falso?" era introdotto dalla lettura di due storie verosimili a finale aperto, ispirate a notizie di attualità pertinenti al funzionamento delle istituzioni europee (diritti dei migranti approdati sul territorio dell'Unione, procedura di stato d'accusa del Parlamento europeo in caso di gravi violazioni dei valori alla base dell'UE, ecc.). Terminata la lettura di una storia, si chiedeva agli studenti quale fosse, tra le coppie di finali proposti, l'opzione corretta in funzione di quanto essi presumevano fosse previsto dalla vigente normativa comunitaria. Quindi, in base all'opzione ritenuta veritiera, erano sollecitati a prendere posto in corrispondenza di un lato o l'altro dello spazio-aula. Si formavano pertanto due squadre omogenee, ciascuna delle quali doveva spiegare all'altra le ragione della propria decisione. Era possibile che, argomentando in modo persuasivo, qualcuno si spostasse da un fronte all'altro. Nel caso in cui tutta la classe, ad eccezione di un solo alunno, si fosse schierata da una parte, il formatore aveva il compito di dialettizzare tra l'esigua minoranza e la stragrande maggioranza. Al termine, illustrava all'attenzione dei ragazzi la risposta corretta ricorrendo ogni volta alla documentazione specifica (per esempio, nella prima storia, che richiamava questioni inerenti ai diritti dei migranti, commentava il regolamento di Dublino III in materia di accoglienza ai rifugiati).

Nel quiz "Io, cittadino che son io?" la classe veniva suddivisa in squadre composte da 4 o 5 studenti ciascuna. Ad ogni squadra veniva consegnato lo stesso quiz a risposta multipla costituito da una decina di domande sui diritti e le responsabilità dei cittadini europei di tutti gli Stati. Terminato il tempo a disposizione (generalmente 15 minuti), il formatore affidava la correzione dei quiz al suo assistente e procedeva giustificando ogni risposta con il supporto di slide, Powerpoint e video. La squadra che aveva ottenuto il punteggio più elevato riceveva un piccolo gadget da condividere con la classe. I quesiti posti offrivano l'occasione per riflettere su temi di natura storica, amministrativa o semplicemente tecnico-pratica di solito poco noti anche a chi, pur essendo cittadino europeo, abbia occasione di spostarsi con frequenza all'interno dell'Unione[19]. 


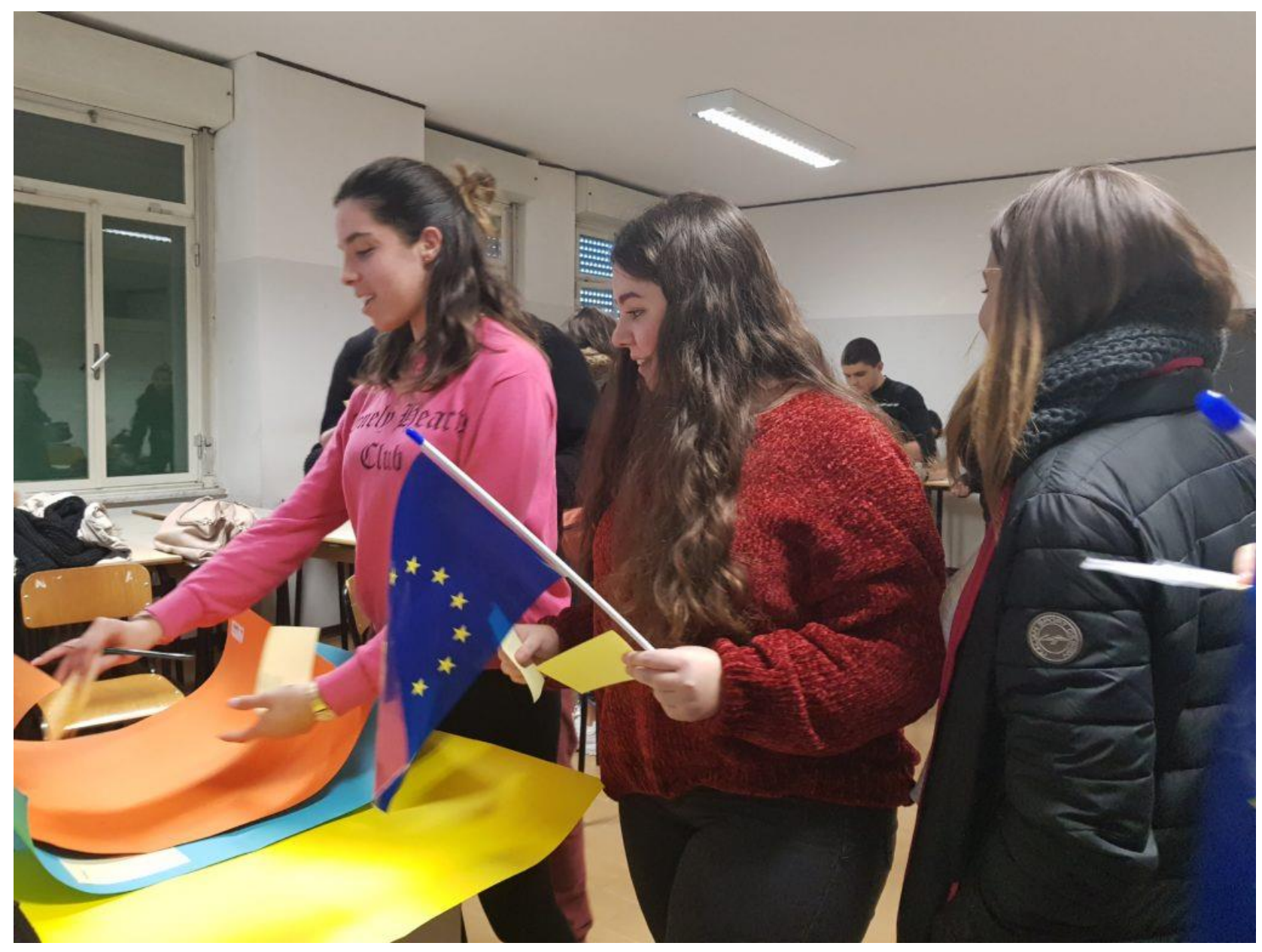

\section{Metodologia}

La finalità primaria del progetto è stata di fornire una conoscenza di base degli elementi costitutivi delle istituzioni dell'UE per far maturare atteggiamenti collaborativi consapevoli e indurre a riflettere sulle opportunità di studio e lavoro di cui i cittadini europei possono fruire[20]. Per questo motivo, si è cercato di ottimizzare l'efficacia del percorso facendo uso di strategie diversificate che, in generale, poggiavano su due modalità didattiche di base. Da un lato, si è fatto ricorso all'approccio presente-passato-presente: partendo da una ricognizione delle opinioni sull'Europa più diffuse tra i giovani e da fatti, luoghi o personaggi tratti dalla cronaca, si è aperto un percorso a ritroso che, appellandosi alla storia passata, ha dato ragione dell'oggi. Dall'altro, si è coniugato l'asse storico-civico con la didattica ludica che ha fatto leva sulla motivazione intrinseca degli studenti. "Imparando in forma di gioco", lo studente opera in un contesto privo di tensioni negative, si diverte e, al contempo, sviluppa capacità sociali. La frequente interazione con il formatore e i compagni condiziona il singolo a mediare l'enunciazione autocentrata delle proprie opinioni, attenua forme di competitività e avvia processi di socializzazione più consapevoli.

Nello specifico, ci si è avvalsi di tecniche didattiche volte ad attualizzare l'esperienza di apprendimento senza mai precluderne gli aspetti emotivi. A tale proposito, la discussione guidata ha perseguito innanzitutto lo scopo di coinvolgere direttamente il gruppo classe e di far emergere qualora fosse opportuno - le dinamiche socio-affettive o il radicamento socio-culturale e familiare che sovente sostanzia nei singoli percezioni confuse o acritiche dichiarazioni di appartenenza. In questo senso, il riferimento orientativo di fondo è stato il cooperative learning (apprendimento cooperativo per lo sviluppo integrato di competenze cognitive, operative e relazionali). 


\section{Obiettivi raggiunti}

Il progetto, apprezzato a larghissima maggioranza dai docenti e dagli studenti, ha soddisfatto gli obiettivi fissati. Esaminando le centinaia di commenti riportate dalle ragazze e dai ragazzi sui postit al termine delle attività, alla voce "cosa mi è piaciuto" i formatori trovavano lunghi elenchi che coprivano per intero le azioni formative proposte. Tra le osservazioni relative a "cosa cambierei" si confrontavano con la richiesta di incrementare la discussione guidata, la visione di video sui principali organismi dell'Unione europea e le occasioni per imparare giocando, che gli studenti avrebbero voluto estendere anche alle materie del curricolo. Assai rari risultavano invece i post-it in corrispondenza della voce "cosa non mi è piaciuto". Tra i quasi duemila partecipanti, i giudizi fortemente negativi non raggiungevano le due decine e spesso erano stati scritti da studenti di classi il cui insegnante referente o non aveva assistito allo svolgimento dei moduli o manifestava aperta sfiducia sui contenuti del progetto. In altri casi, valutazioni con riserva venivano avanzate in base a scetticismi pregressi sull'efficacia delle istituzioni dell'Unione europea e "nonostante" l'apprezzamento dell'azione didattica. Di fatto, le eventuali criticità riguardavano la breve durata del progetto e si traducevano nella richiesta di ripeterlo per approfondirne la complessità.

\section{Conclusioni}

In sintesi, proviamo a riassumere gli elementi che hanno contribuito a soddisfare gli obiettivi posti.

La destrutturazione dello spazio-aula - sollecitata dalla disposizione in cerchio della classe per lo svolgimento dell'attività "Ice breaker" - ha incuriosito e provocato un iniziale disorientamento, ma si è poi rivelata un fattore chiave per la creazione di un clima dialogico costruttivo e per l'elaborazione di idee più autonome e consapevoli in situazioni di gruppo (tutti i presenti sono stati sollecitati ad intervenire "alla pari" e ad "ascoltarsi").

La conoscenza, spesso approssimativa, che i giovani hanno della storia, delle istituzioni e delle funzioni dell'Unione europea, messa in gioco tramite il dibattito "Questo o quello?", ha introdotto il role playing in funzione didattica. Chiamati in causa singolarmente, $\mathrm{i}$ ragazzi hanno risposto con maggiore responsabilità. È così emerso un interesse nuovo per le potenzialità che la cittadinanza, vissuta consapevolmente, è in grado di offrire.

La risoluzione a squadre del quiz "Io, cittadino che sono io?", con la discussione delle risposte e la premiazione finale, nata dal proposito di realizzare il cooperative learning in forma ludica, ha sollecitato un virtuoso processo di competizione e ha confermato il valore aggiunto proprio delle azioni svolte insieme agli altri.

Ad un esito analogo hanno portato il brainstorming e la strategia didattica dell'incident che, con minime forzature, hanno sorretto l'ideazione dell'attività denominata "Vero o falso?". L'esito delle discussioni (quella, per esempio, sul concetto di "democrazie illiberali" o di "sovranità statale" in Europa), suffragate da brevi studi di caso[21], ha contenuto la tendenza a sentenziare mediante scorciatoie semplicistiche, agevolando il dialogo e la consapevolezza della complessità.

Ha pertanto trovato conferma l'idea-guida del progetto ossia che, per educare con efficacia alla cittadinanza democratica e - in particolare - a quella europea, il nesso didattico tra contenuti, variabili cognitive e dinamiche psicologiche è imprescindibile. 
Note:

[1] Questa immagine, realizzata dal vignettista Walter Leoni, è stata utilizzata, insieme a molte altre, nell' "Ice breaker", il modulo iniziale del progetto didattico "Memoria storica, cittadinanza, innovazione civica. Progetto per le nuove generazioni" per la cui descrizione si rimanda ai successivi paragrafi.

[2] Nell'agile saggio, fresco di stampa, Nazionalisti e patrioti (Roma-Bari, Laterza, 2019), Maurizio Viroli traccia la distinzione tra nazionalismo e patriottismo: prendendo le mosse da Herder e giungendo sino ai nostri giorni, l'autore delinea la radicale differenza tra questi due ideali che, pur suscitando forti passioni e appellandosi a un comune sentire, si richiamano a principi, valori e prassi politiche opposte.

[3] Per una storia del processo di unificazione europea cfr. U. Morelli, Storia dell'integrazione europea, Milano, Guerini e Associati, 2011. Un'analisi ad ampio raggio degli elementi costitutivi della storia europea è contenuta in A. Cavalli, A. Martinelli, La società europea, Bologna, il Mulino, 2015.

[4] Sul tema, quanto mai dibattuto e controverso, dell'identità rimando alle acute riflessioni svolte dall'antropologo Francesco Remotti in L'ossessione identitaria (Roma-Bari, Laterza, 2010) e Somiglianze. Una via per la convivenza (Roma-Bari, Laterza, 2019). Per una disamina critica della concezione identitaria dell'Europa cfr. P. Rossi, L'identità dell'Europa, Bologna, il Mulino, 2007.

[5] In merito alla decostruzione della supposta "naturalità" delle nazioni cfr. A.M. Thiesse, La creazione delle identità nazionali in Europa, Bologna, il Mulino, 2001, in cui l'autrice ricostruisce $\mathrm{i}$ processi mediante i quali un'élite intellettuale si è adoperata con passione per creare, nei secoli XVIII e XIX, un'identità nazionale e plasmare a fondo l'immaginario collettivo.

[6] Su queste problematiche a livello didattico può essere utile P. Battifora, Alla ricerca dell'identità europea. Strumenti concettuali, storia e un dossier didattico, realizzato in occasione della Summer School 2017 (Insegnare l'Europa contemporanea. Politiche, culture, società: dalla storia al tempo presente) e inserito nel dossier Insegnare l'Europa contemporanea, curato da E. Pagano e P. Battifora, che raccoglie le relazioni e i materiali del corso (Novecento.org, n. 9, febbraio 2018).

[7] Palazzo Ducale Fondazione per la Cultura, Accademia Ligustica di Belle Arti, Gioventù Federalista Europea Genova, Europe Direct Genova. Il progetto, per taluni specifici eventi, ha ottenuto il patrocinio del Comune di Genova e della Città Metropolitana di Genova e si è avvalso di un contributo finanziario della Compagnia di San Paolo.

[8] In ambito universitario 290 sono stati gli studenti dell'ateneo genovese che hanno partecipato al progetto dell'ILSREC e per i quali sono stati approntati specifici moduli didattici.

[9] L'ILSREC ha collaborato alla realizzazione del convegno, organizzato dalla Scuola di Scienze Sociali dell'Università di Genova e svoltosi dal 6 all' 8 marzo.

[10] Allestita negli spazi di Palazzo Ducale dal 19 al 25 marzo e visitata da oltre 700 persone, la mostra ha esposto quindici opere selezionate dalla giuria del concorso d'arte, promosso dall'ILSREC e rivolto ai giovani artisti (cfr. www.ilsrec.it/cittadinanza-europea/bando-cittadinanzaeuropea/). La cerimonia di premiazione dei vincitori si è svolta il 21 marzo a Palazzo Ducale. 
[11] Il numero monografico della rivista, presentato il 26 marzo, contiene saggi e interventi di autorevoli politici e studiosi, tra cui Giorgio Napolitano, Romano Prodi, Mario Draghi, Giuliano Amato, Emma Bonino, Alessandro Cavalli, Daniela Preda, Giovanni Maria Flick, Sergio Cofferati (cfr. www.ilsrec.it/storia-e-memoria-2-2019-dove-va-leuropa/).

[12] Il 22 gennaio si è tenuta a Palazzo Tursi la presentazione pubblica del progetto didattico; il 29 marzo si è svolta all'Università di Genova la giornata di studi federalisti, in occasione della quale è stata realizzata la mostra, curata da Angelica Radicchi, "I volti dell'Europa" (cfr. "Storia e memoria", n. 2/2019, p. 169); il 10, 16 e 17 aprile sono stati organizzati incontri, rivolti alla società civile, sul tema "Unione europea: istruzioni per l'uso"; il 30 maggio, a Palazzo Doria-Spinola, il giurista Vincenzo Roppo ha svolto la lectio magistralis dal titolo "La democrazia italiana nell'ordinamento europeo" (la videoregistrazione dell'intervento è disponibile sul canale YouTube dell'ILSREC, https://www.youtube.com/watch?v=3cnnWVSe_Kg): tale iniziativa, realizzata in sinergia con la Prefettura di Genova in prossimità della Festa della Repubblica del 2 giugno, si è configurata quale evento conclusivo del progetto. In occasione di tale prolusione è stata allestita, presso il loggiato di Palazzo Doria-Spinola, la mostra "Costituzione, cittadinanza europea, patto di convivenza e storia del presente", ideata da Giosiana Carrara (cfr. "Storia e memoria", n. 2/2019, pp. 170-71).

[13] La videoregistrazione della conferenza di Marcello Flores è disponibile sul canale Youtube dell'ILSREC (https://www.youtube.com/watch?v=h7f8OyUX4ug).

[14] I formatori dello staff dell'ILSREC, impegnati nel progetto, per la provincia di Genova sono stati: Paolo Battifora, Francesco Caorsi, Donatella Chiapponi, Silvia Giulini, Alessio Parisi e Angelica Radicchi; per la provincia di Savona: Giosiana Carrara e Angelica Radicchi; per la provincia di Imperia: Silvia Giulini e Angelica Radicchi.

[15] Com'è noto, circa 400 milioni di cittadini membri dell'Unione hanno partecipato alle elezioni del Parlamento europeo tenutesi tra il 23 e il 26 maggio 2019. In Italia si è votato il 26 maggio e l'affluenza alle urne si è attestata sul 54,50 \% (cfr. https://risultati-elezioni.eu/affluenza/).

[16] Particolarmente utili, a tale riguardo, alcuni video di repertorio come quello sulla firma dei Trattati di Roma (cfr. https://www.youtube.com/watch?v=s4nXDadjlrE).

[17] Cfr. l'efficace video di spiegazione del Parlamento europeo all'url https://www.youtube.com/watch?v=82Rphls00D0.

[18] Cfr. Documenti e pubblicazioni per insegnanti sul sito ufficiale dell'Unione europea (https://europa.eu/european-union/documents-publications/teachers_it) e l'opuscolo L'unione europea che cos'è e che cosa fa scaricabile all'url http://publications.europa.eu/webpub/com/euwhat-it-is/it/.

[19] Per esempio, tra le domande del quiz si poneva il caso di una giovane francese che, vittima di un infortunio in Italia, avesse o meno diritto a ricevere l'assistenza sanitaria nel nostro Paese. La risposta corretta, ossia che la giovane - mostrando il suo tesserino sanitario europeo - poteva essere curata in Italia e rimborsata in seguito del costo della prestazione, veniva documentata anche tramite il video https://europa.eu/european-union/life/healthcare_it.

[20] Una modalità per affrontare questo aspetto e mostrare concretamente ai giovani che cosa l'Europa può fare per loro può essere colta a partire dalla visione del portale What Europe does for $m e$ (https://what-europe-does-for-me.eu/it/portal) che, non a caso, ha riscosso particolare interesse. 
[21] Per esemplificare, richiamiamo tra le tante una domanda del quiz che si riferiva alla particolare situazione storico-politica di Cipro, la cui capitale - Nicosia - è l'unica in Europa ad essere ancora separata dalla Green line nelle due parti turca e greca. La realtà di Cipro incuriosiva gli studenti che chiedevano le cause della persistenza di questo "muro". Per approfondire l'argomento, si fornivano rapidi cenni alle fonti, integrandole con una scheda sintetica sulla storia dell'invasione turca del 1974 e la visione di passaggi dal video Vivere separati a Cipro, reportage dall'ultima capitale divisa d'Europa (https://www.youtube.com/watch?v=CRv8-18DJ0I). Talora, su richiesta, si suggeriva ai docenti di programmare nelle classi un'analisi specifica della questione cipriota tramite lo strumento didattico dello studio di caso, rimandando per informazioni più dettagliate all'articolo Gli studi di caso. Insegnare storia in modo partecipato e facile di Antonio Brusa (Novecento.org, $\mathrm{n}$. 3, dicembre 2014). 